\title{
Prevalencia de cronicidad y multimorbilidad en la población cubierta por el servicio público de teleasistencia de Euskadi: impacto en costes y calidad de la atención
}

\section{Prevalence and costs of chronicity and multimorbidity in the population covered by the Basque public telecare service}

\author{
J.F. Orueta ${ }^{1}$, E. Alonso-Morán ${ }^{2}$, R. Nuño-Solinis ${ }^{2}$, A. Alday-Jurado ${ }^{3}$, E. Gutiérrez-Fraile ${ }^{3}$, \\ A. García-Álvarez ${ }^{2}$
}

\section{RESUMEN}

Fundamento. La teleasistencia pública en Euskadi (betiON) es un servicio ofrecido por el Departamento de Empleo y Políticas Sociales del Gobierno Vasco que proporciona atención telefónica a las poblaciones dependientes y de mayor edad, ofreciéndoles comunicación con un centro de atención. Su conexión con dispositivos asistenciales sanitarios permite disponer de sistemas de información que comparten datos de índole sanitaria y social, lo cual posibilita una caracterización de la población atendida y el desarrollo de modelos de provisión integrada social y sanitaria. Objetivo. Describir la distribución de enfermedades crónicas y multimorbilidad entre los usuarios de betiON, estimar el coste de su atención sanitaria, calcular las hospitalizaciones evitables y comparar tales indicadores con la población general.

Métodos. Se extrajo información de las bases de datos sanitarias públicas y de betiON. Se caracterizó la población de teleasistencia mayor de 65 años de edad $(\mathrm{N}=23.742)$ respecto a variables demográficas, socio-económicas, carga de morbilidad, costes y hospitalizaciones evitables. Se realizaron ajustes indirectos por edad y sexo para comparar la prevalencia de enfermedades con la población general y modelos de regresión para coste y hospitalizaciones evitables.

Resultados. En la población de teleasistencia, el promedio de edad es 83 años, el $80 \%$ son mujeres, un $55,1 \%$ viven solos y residen en zonas más deprimidas. El 42,5\% de los varones y $36,5 \%$ de las mujeres está afectado por 5 o más patologías crónicas, siendo 4,06 el promedio de enfermedades por persona. Además los costes de su atención sanitaria son más elevados que los de la población general (57\%). Ajustando por edad, sexo y morbilidad, no existen diferencias en la tasa de hospitalizaciones evitables con la población general.

Conclusiones. Los usuarios de teleasistencia presentan mayor carga de morbilidad, mayor necesidad de atención y condiciones sociales más desfavorables, pese a lo cual la calidad de la atención recibida es equiparable a la población general. La coordinación de servicios sociales y sanitarios a través de la conexión de sistemas de información y la teleasistencia como plataforma de servicios ofrecen oportunidades relevantes de innovación en la intervención sobre esta población.

Palabras clave. Multimorbilidad. Enfermedades crónicas. Teleasistencia. Cronicidad. Costes de atención.

\begin{abstract}
Background. The public telecare service in Euskadi (betiON) is a service offered by the Department of Employment and Social Policy of the Basque Government and offers telephone support to elderly and disabled populations, providing communication with an assistance centre. This connection with health care devices allows implementing shared information systems with health and social data, which enable a characterization of the attended population and the development of integrated models for health and social provision.

Objective. To describe the distribution of chronic diseases and multimorbidity among users of betiON, to estimate the cost of their health care and to compare these indicators with those of the general population.

Methods. We extracted information from betiON and public healthcare databases. We characterized telecare population above 65 years $(\mathrm{N}=23.742)$ and compared it with the total Euskadi one $(\mathrm{N}=2.262 .707)$ with respect to demographic, socio-economic burden, costs and ambulatory care sensitive conditions.
\end{abstract}

Results. In the telecare population, the average age is 83 years $80 \%$ are women, $55,1 \%$ live alone and dwell in poorer neighborhoods. A $42,5 \%$ of males and $36,5 \%$ of women are affected by five or more chronic conditions, averaging 4,06 pathologies per person. Also they exhibit higher healthcare costs than the general population.

Conclusions. Telecare users have a higher disease burden, greater need for healthcare and unfavorable social conditions. Telecare provides an opportunity for innovation and intervention, through coordination of social and healthcare services.

Key words. Multimorbidity. Chronic diseases. Telehealth. Chronicity. Health care costs.

\section{An. Sist. Sanit. Navar. 2013; 36 (3): $429-440$}

1. Osakidetza, Servicio Vasco de Salud.

2. O+Berri, Instituto Vasco de Innovación Sanitaria. Fundación Vasca de Innovación e Investigación Sanitaria.

3. Osatek, S.A. Sociedad Pública del Gobierno Vasco.

Recepción: 3 de mayo de 2013

Aceptación provisional: 19 de junio de 2013

Aceptación definitiva: 4 de julio de 2013

\section{Correspondencia:}

Juan F. Orueta

Centro de Salud Astrabudua

Mezo, 35

48950 Erandio (Bizkaia) 


\section{INTRODUCCIÓN}

Desde hace varias décadas la dinámica demográfica en todo el mundo se caracteriza por un descenso de la mortalidad y una disminución de la fecundidad ${ }^{1}$.Estos fenómenos han provocado profundos cambios en la estructura de la población en prácticamente todos los países y el envejecimiento poblacional supone un reto desde el punto de vista económico, social y sanitario. Un grupo que genera especial preocupación son los ancianos que viven solos, pues se encuentran en mayor riesgo de sufrir aislamiento social y privación económica, y en consecuencia, pueden requerir el apoyo de intervenciones especiales.

En nuestro medio, estos hechos también se observan y de una forma especialmente notoria. El colectivo de personas mayores de 65 años, en el País Vasco, supone actualmente el 19,2\% de la población total (frente al 16,9\% del conjunto de España y $7,5 \%$ de todo el mundo) y se constata un sobreenvejecimiento de la población anciana (las personas de 80 y más años suponen ya el $5,9 \%$ de la población total y el $29,3 \%$ de los mayores) ${ }^{2}$. Además, según las proyecciones del Instituto Vasco de Estadística (EUSTAT), esta tendencia se mantendrá en las próximas décadas ${ }^{3}$ y se augura que, para 2050, el grupo de mayores de 65 años doblará su tamaño en términos relativos, llegando a suponer el $38,8 \%$ de la población total.

Las limitaciones físicas, psicológicas y funcionales inherentes al proceso de envejecimiento obligan a los sistemas de prestación de cuidados a adaptarse a esta nueva situación. Desde el punto de vista sanitario, la presencia de enfermedades crónicas en mayores de 65 años es sumamente frecuente y la coexistencia de múltiples patologías en una misma persona es un hallazgo tan común que actualmente se considera que la multimorbilidad (concurrencia de dos o más patologías crónicas en un paciente) es la condición crónica más prevalente en este grupo etario ${ }^{4}$. Así, a nivel internacional, la prevalencia de multimorbilidad está siendo estudiada en población general y en colectivos específicos en función de su edad o por ser atendidos en determinados dispositivos asistenciales ${ }^{5-10}$. Pese a no existir un consenso sobre cómo medir estas prevalencias ${ }^{11}$, sí se reconoce la necesidad de profundizar en su medición y en la identificación de perfiles y combinaciones determinadas de patologías. También su impacto en mortalidad, discapacidad y calidad de vida, así como en costes y utilización de servicios sanitarios está siendo investigado cada vez más ${ }^{12-15}$.

En este contexto, se estima que en Euskadi un $22,8 \%$ de las personas de 65 o más años necesitan ayuda para alguna de las actividades de la vida diaria y un $17,2 \%$ la requieren para llevar a cabo las actividades básicas ${ }^{16}$. Además, los cambios en la estructura familiar también afectan a la población anciana y se comprueba, siguiendo la tendencia europea, un creciente número de los hogares unipersonales de personas mayores. Así, en Euskadi se ha pasado de un $16,5 \%$ en 1993 a un $24,8 \%$ en $2010^{16}$.

En Euskadi, el servicio público de teleasistencia (betiON) dependiente del Departamento de Empleo y Políticas Sociales se fortalece con una línea de cooperación con el Departamento de Salud, con la aspiración de facilitar la integración de las personas en su entorno familiar y social habitual, evitando institucionalizaciones innecesarias. Se enmarca en un esquema de asistencia integral centrada en la persona, cuyos objetivos son ofrecer a la ciudadanía canales de interacción no presenciales, con funcionamiento $24 \times 7$, potenciar modelos de atención y cuidados innovadores en el domicilio, promover estilos de vida más saludables y realizar un seguimiento a distancia de personas en situaciones especiales, identificando riesgos y actuando proactivamente.

El servicio betiON está circunscrito a la Comunidad Autónoma del País Vasco y son considerados destinatarios los ciudadanos con los siguientes perfiles sociales: personas que presenten discapacidad intelectual, física, sensorial o que padezcan una enfermedad mental y se hallen en situación de dependencia; personas con riesgo de aislamiento social; los mayores 
de 65 años dependientes y los mayores de 75 años que vivan solos. La resolución del expediente administrativo de solicitud se notifica en un plazo máximo de dos meses. Los servicios sociales de base de los ayuntamientos, así como los médicos de atención primaria de la red de Osakidetza son los principales agentes en la difusión del servicio. La historia de la Teleasistencia ha estado ligada a la dependencia y a la discapacidad. El Gobierno Vasco ha incorporado el criterio de soledad para las personas mayores de 75 años, con el fin de favorecer la permanencia en su entorno habitual. El decreto 144/2011, de 28 de junio, regula todos los aspectos concernientes al servicio público de Teleasistencia que deben contenerse en la Cartera de Prestaciones y Servicios del Sistema Vasco de Servicios Sociales.

Con el fin de profundizar nuestro conocimiento sobre las necesidades de las personas incluidas en el servicio público de teleasistencia de Euskadi se planteó este estudio cuyo objetivo es describir la distribución de enfermedades crónicas y multimorbilidad entre los usuarios del servicio, estimar el coste de su atención sanitaria, conocer la calidad de la atención que reciben a través de las hospitalizaciones evitables y comparar tales indicadores con los de la población general de Euskadi.

\section{MATERIAL Y MÉTODOS}

Este es un estudio descriptivo, para el cual se utilizó la base de datos del programa de estratificación poblacional (PREST) del servicio vasco de salud. El programa de estratificación en el País Vasco comenzó en el año 2010 y su objetivo es clasificar a todos los ciudadanos de la comunidad autónoma en función de sus futuras necesidades asistenciales, combinando diferentes fuentes de información para obtener las siguientes variables: diagnósticos (de atención primaria, urgencias y de altas de hospital), prescripciones, procedimientos y coste de la atención ${ }^{17}$. Con dicha información, los ciudadanos de País Vasco son clasificados anualmente empleando el sis- tema de case-mix Adjusted Clinical Groups (ACGs), lo que permite identificar los problemas de salud que presentan.

El total de personas cubiertas por el servicio de teleasistencia, a 1 de septiembre de 2011, eran 25.707 ciudadanos. Dado que, en el País Vasco, los servicios de salud y los servicios sociales emplean identificadores diferentes para las personas, sólo en 24.439 ciudadanos $(95,1 \%)$ resultó factible vincular ambos identificadores y acceder a su información contenida en la base de datos de estratificación. De este último conjunto, se excluyeron 615 personas $(2,5 \%)$ ya que su edad era inferior a 65 años, por lo que la población de estudio corresponde a 23.742 usuarios del servicio betiON. Cada usuario de betiON firmó una autorización para que el servicio de teleasistencia pudiera vincular su información con la de las bases de datos de Osakidetza.

El periodo de seguimiento fue de 12 meses (de 1 de septiembre de 2011 a 31 de agosto de 2012). Se registraron al inicio de estudio las variables demográficas (edad y sexo), enfermedades crónicas registradas e índice de privación del área de residencia. Durante los siguientes 12 meses se observó la presencia de hospitalizaciones evitables mediante una atención ambulatoria oportuna (ambulatory care sensitive conditions [ACSC]), el coste de la atención sanitaria y, para ajustar la morbilidad al hacer comparaciones respecto al mismo, se recurrió a información obtenida a partir del empleo del sistema ACG.

Para detectar la presencia de enfermedades crónicas se revisaron los datos de diagnósticos y prescripciones durante los 4 últimos años de cada una de las personas incluidas y se adoptó una metodología similar a la descrita por otros autores ${ }^{14}$. Se definió un listado de 52 enfermedades y se acordaron criterios específicos en cada una de ellas para ser considerada como activa. Una descripción de este proceso se incluye como material suplementario. Se consideró multimorbilidad la coexistencia de dos o más enfermedades en la misma persona.

El índice de privación de la sección censal de residencia se empleó como indicador 
social. Una sección es la unidad territorial más pequeña en la que puede desagregarse la información del censo de población y se define fundamentalmente por criterios de volumen de población, delimitándose además por accidentes geográficos o urbanísticos del territorio. Si bien el número de habitantes de cada una de ellas es variable, la mediana de población por sección censal es de 1.200 habitantes. Su tamaño reducido favorece la homogeneidad de las viviendas que la componen. Este índice se construyó a partir de indicadores relativos a trabajo (desempleo, trabajadores manuales y eventuales) y educación (instrucción insuficiente total y en jóvenes) ${ }^{19}$. Para este estudio, el índice de privación fue categorizado en quintiles, representando el uno la zona de menor privación social y la cinco la de mayor privación.

Para establecer la presencia de hospitalizaciones evitables se recurrió al listado elaborado por Caminal y otros ${ }^{18}$. Se consideró que una persona presentaba dichas hospitalizaciones si constaban en el CMBD en los últimos 12 meses uno o más ingresos atribuibles a estos motivos ${ }^{20}$.

Respecto al coste, éste pudo obtenerse directamente para las prescripciones de atención primaria registradas en las historias clínicas informatizadas. En los otros casos (visitas a urgencias de hospital, sesiones de rehabilitación, consultas externas de atención especializada, médicos y enfermeras de atención primaria; analíticas y exploraciones radiológicas solicitadas en atención primaria; algunos procedimientos como diálisis, radioterapia o quimioterapia realizados en hospital de día) se multiplicó el número de servicios a cada paciente por su coste estandarizado.

Los costes de hospitalizaciones y procedimientos de cirugía mayor ambulatoria se calcularon en función de los pesos de sus correspondientes Grupos Relacionados por el Diagnóstico (GRD) ${ }^{20}$. De la estimación de coste quedaron excluidos algunos servicios de los que no fue posible obtener información: ingresos de hospitales psiquiátricos, servicios de hospitali- zación a domicilio y hospital de día (excepto los procedimientos anteriormente citados), transporte sanitario, prótesis y otros equipos entregados a pacientes en domicilio.

Para ajustar el coste en función de la morbilidad se recurrió clasificar los diagnósticos asignados a los pacientes en los 12 meses del periodo de observación en Aggregated Diagnosis Groups (ADGs). Los ADGs son 32 grupos de diagnósticos que genera el sistema de case-mix ACGs ${ }^{21}$, en función de la probabilidad de recurrencia o persistencia de ese problema de salud y el consumo de recursos esperado para su tratamiento. Se considera que un paciente presenta un determinado ADG si alguno de sus diagnósticos está incluido en este grupo.

Se realizaron ajustes indirectos por edad y sexo (aplicando a la población betiON los promedios observados en la población general) para establecer comparaciones en las prevalencias de enfermedades crónicas. Como métodos estadísticos para establecer las odds ratio para hospitalizaciones evitables se emplearon análisis de regresión logística. Para obtener los valores esperados de coste de los recursos sanitarios consumidos se emplearon modelos de regresión lineal múltiple realizando dos tipos de ajuste por edad y sexo; y por edad, sexo, índice de privación y los 32 ADGs como variables dummy. Se utilizaron pruebas no paramétricas (Wilcoxon Test) para analizar las diferencias entre sexos y (Wilcoxon Signed Rank Test) entre valores observados y esperados. El software utilizado para los cálculos estadísticos fue SAS 9.2.

\section{RESULTADOS}

La tabla 1 presenta la distribución de la población de estudio en grupos de edad, sexo y unidad de convivencia. De las 23.724 personas observadas, la edad media es 83 años y un $80 \%$ son mujeres. El porcentaje de varones que viven solos es $38 \%$ y $59 \%$ en mujeres. 
PREVALENCIA DE CRONICIDAD Y MULTIMORBILIDAD EN LA POBLACIÓN CUBIERTA POR EL SERVICIO PÚBLICO...

Tabla 1. Población incluida en el Servicio Público de Teleasistencia de Euskadi (betiON). Distribución en grupos de edad y unidad de convivencia. Promedio de enfermedades crónicas y comparación con población total de población asignada al Servicio Público de Salud (Osakidetza) País Vasco

\begin{tabular}{|c|c|c|c|c|c|c|c|}
\hline \multirow{2}{*}{$\begin{array}{l}\text { Grupos } \\
\text { de edad }\end{array}$} & \multirow{2}{*}{$\mathbf{N}$} & \multicolumn{4}{|c|}{ Unidad de convivencia } & \multicolumn{2}{|c|}{$\begin{array}{c}\text { Promedio de } \\
\text { Enfermedades Crónicas }\end{array}$} \\
\hline & & No consta & Vive solo & $\begin{array}{l}\text { Vive con } 1 \\
\text { persona }\end{array}$ & $\begin{array}{l}\text { Vive con } 2 \text { ó } \\
\text { más personas }\end{array}$ & betiON & Osakidetza \\
\hline \multicolumn{8}{|l|}{ Varones } \\
\hline $65-69$ & 127 & $15(11,6 \%)$ & $60(46,6 \%)$ & $38(34,9 \%)$ & $14(6,8 \%)$ & 4,26 & 2,03 \\
\hline $70-74$ & 254 & $40(15,8 \%)$ & $104(41,6 \%)$ & $96(37,1 \%)$ & $14(5,5 \%)$ & 4,42 & 2,49 \\
\hline $75-79$ & 723 & $112(16,1 \%)$ & $314(43,0 \%)$ & $251(34,2 \%)$ & $46(6,7 \%)$ & 4,47 & 2,98 \\
\hline $80-84$ & 1.470 & $196(13,4 \%)$ & $552(36,9 \%)$ & $617(42,6 \%)$ & $105(7,0 \%)$ & 4,53 & 3,34 \\
\hline $85+$ & 2.046 & $257(12,5 \%)$ & $750(36,4 \%)$ & $875(42,9 \%)$ & $164(8,2 \%)$ & 4,24 & 3,18 \\
\hline Total & 4.620 & $620(13,9 \%)$ & $1.780(38,4 \%)$ & $1.877(39,8 \%)$ & $343(7,8 \%)$ & 4,38 & 2,67 \\
\hline \multicolumn{8}{|l|}{ Mujeres } \\
\hline $65-69$ & 321 & $36(12,4 \%)$ & $177(57,2 \%)$ & $87(24,6 \%)$ & $21(5,8 \%)$ & 4,40 & 1,97 \\
\hline $70-74$ & 832 & $111(12,6 \%)$ & $478(57,7 \%)$ & $202(25,4 \%)$ & $41(4,3 \%)$ & 4,13 & 2,39 \\
\hline $75-79$ & 2.956 & $372(12,4 \%)$ & $1.791(60,9 \%)$ & $655(22,2 \%)$ & $138(4,4 \%)$ & 4,20 & 2,85 \\
\hline $80-84$ & 6.144 & $662(10,8 \%)$ & $3.846(62,4 \%)$ & $1.383(22,6 \%)$ & $253(4,2 \%)$ & 4,17 & 3,20 \\
\hline $85+$ & 8.851 & $971(10,9 \%)$ & $4.994(55,6 \%)$ & $2.375(27,3 \%)$ & $511(6,1 \%)$ & 3,82 & 2,99 \\
\hline Total & 19.104 & $2.152(11,3 \%)$ & $11.286(58,6 \%)$ & $4.702(24,8 \%)$ & $964(5,2 \%)$ & 4,01 & 2,64 \\
\hline
\end{tabular}

Como cabía esperar, las enfermedades crónicas son más prevalentes en la población betiON que en el conjunto de ciudadanos atendidos en Osakidetza. Por otra parte, es conocida la relación entre envejecimiento y multimorbilidad y así se comprueba en la población general: los promedios de enfermedades crónicas van aumentando con la edad hasta alcanzar los 85 años; sin embargo, los usuarios de betiON muestra un patrón diferente, ya que las diferencias en los promedios de los grupos de edad son pequeñas y no se observa dicha progresión.

En la población estudiada, la multimorbilidad es tan habitual que el $42,5 \%$ de los varones y $36,5 \%$ de las mujeres está afectado por 5 o más enfermedades crónicas (Fig. 1). En ambos sexos la hipertensión arterial y diabetes mellitus son muy frecuentes (Tabla 2), pero en otros casos son patentes las diferencias: mientras que en el sexo masculino predominan las enfermedades cardiovasculares y la enfermedad pulmonar obstructiva crónica (EPOC) en el femenino lo hacen las enfermedades osteoarticulares y trastornos psiquiátricos.

El servicio de teleasistencia incluye al $5,3 \%$ de la población total de mayores de 65 años del País Vasco mayores de 65 años y al $12 \%$ de mayores de 80 años. En relación con los factores socioeconómicos, se comprueba que las coberturas son más altas en las poblaciones más desfavorecidas y este gradiente es marcado en ambos sexos (Tabla 3). En todos los casos la cobertura del servicio en mujeres supera en más de dos veces la de los varones.

El porcentaje de usuarios betiON ingresados por ACSC fue 6,94\%, más del doble de la población total de mayores de 65 años del País Vasco, y la probabilidad de presentar algún ingreso por estos motivos fue más alta en varones que en mujeres. Tras 


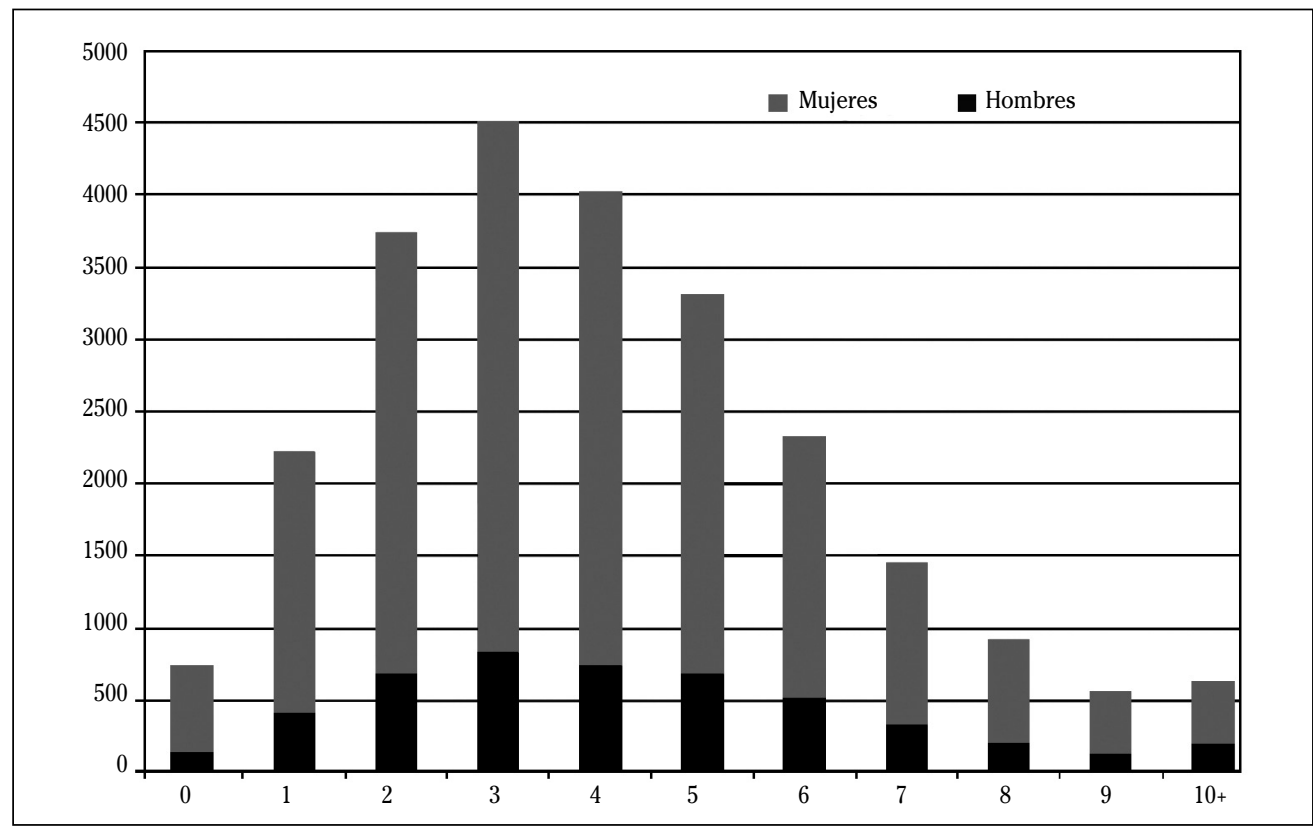

Figura1. Distribución de la población en el Servicio Público de Teleasistencia de Euskadi (betiON) en función del número de enfermedades crónicas.

ajustar la morbilidad (mediante ADGs), no se encontraron deferencias entre los usuarios de teleasistencia y el resto, aunque se mantuvieron las diferencias entre sexos en ambos grupos (Tabla 4).

En la tabla 5 se presentan los promedios de coste, comparándose los valores observados con los esperados, empleando dos ajustes: solamente por edad y sexo en un caso y añadiendo una medida de la morbilidad en el segundo. Los promedios de coste en varones son, en todos los casos, más altos que los de las mujeres y estas diferencias son estadísticamente significativas excepto en atención primaria. La población betiON también muestra unos costes más altos de la atención cuando se compara con los presentados por otros pacientes de Osakidetza; esta situación persiste tras ajustar dichos promedios de coste por edad. Sin embargo, al incluir la morbilidad (ADGs) en los modelos, las diferencias en coste total, de atención primaria, prescripciones y especializada sin ingreso disminuyen (aunque las diferencias continúan siendo estadísticamente significativas); en el caso de ingresos hospitalarios, hombres y mujeres se comportan de forma diferente: mientras que los hombres asistidos por betiON también presentan un coste más alto que otros varones, las mujeres incluidas en el programa de teleasistencia utilizan menos los servicios hospitalarios que el resto de la población femenina del País Vasco.

Los coeficientes de determinación de los modelos de regresión lineal múltiple que explicaron los costes de atención primaria, especializada, urgencias, ingresos, prescripciones y coste total a través de las variables independientes ya citadas (edad, sexo, índice de privación y ADGs) fueron 0,5362; 0,$1236 ; 0,2968 ; 0,2586 ; 0,2538$ y 0,3737 respectivamente. El coeficiente de los modelos de regresión logística (Cox and Snell) ${ }^{22}$ para explicar las hospitalizaciones evitables fue 0,0445 en la población general (variables independientes; sexo, edad, ADGs, índice de privación y el hecho de estar incluido en el servicio de teleasistencia) y 0,1474 en la población usuaria del servicio de teleasistencia (variables independientes: sexo, edad, ADGs e índice de privación). 
Tabla 2. Prevalencias de las enfermedades crónicas más frecuentes en la población del Servicio Público de Teleasistencia de Euskadi (betiON). Los valores esperados se han estimado mediante ajuste indirecto por edad y sexo, aplicando los valores obtenidos en la población total de Osakidetza

\begin{tabular}{|c|c|c|c|c|}
\hline \multirow{2}{*}{ Enfermedades } & \multicolumn{2}{|c|}{ Hombres } & \multicolumn{2}{|c|}{ Mujeres } \\
\hline & $\mathrm{N}$ (observados) & $\mathrm{N}$ (esperados) & $\mathrm{N}$ (observados) & $\mathrm{N}$ (esperados) \\
\hline Hipertensión & $77,7 \%$ & $64,8 \%$ & $79,7 \%$ & $68,2 \%$ \\
\hline Diabetes Mellitus & $30,1 \%$ & $21,6 \%$ & $25,5 \%$ & $18,6 \%$ \\
\hline Degeneración articular & $14,2 \%$ & $10,4 \%$ & $28,3 \%$ & $19,1 \%$ \\
\hline Dispepsia & $19,0 \%$ & $13,0 \%$ & $24,1 \%$ & $16,4 \%$ \\
\hline Ansiedad & $12,8 \%$ & $7,5 \%$ & $21,2 \%$ & $14,8 \%$ \\
\hline Fibrilación auricular & $24,1 \%$ & $17,6 \%$ & $16,0 \%$ & $12,5 \%$ \\
\hline Depresión & $13,1 \%$ & $6,6 \%$ & $21,7 \%$ & $13,9 \%$ \\
\hline Neoplasia maligna & $17,2 \%$ & $14,1 \%$ & $8,7 \%$ & $6,9 \%$ \\
\hline Glaucoma & $14,2 \%$ & $10,6 \%$ & $14,3 \%$ & $10,8 \%$ \\
\hline Osteoporosis & $2,3 \%$ & $1,5 \%$ & $18,7 \%$ & $13,2 \%$ \\
\hline Accidente cerebro-vascular & $20,8 \%$ & $12,3 \%$ & $13,6 \%$ & $10,2 \%$ \\
\hline Hipertrofia prostática & $23,1 \%$ & $19,7 \%$ & - & - \\
\hline Enfisema, bronquitis crónica, EPOC & $21,5 \%$ & $14,9 \%$ & $6,7 \%$ & $4,9 \%$ \\
\hline Cardiopatía isquémica & $18,6 \%$ & $12,7 \%$ & $9,0 \%$ & $5,8 \%$ \\
\hline Enfermedad cardiaca crónica, otras & $15,8 \%$ & $10,6 \%$ & $9,6 \%$ & $6,6 \%$ \\
\hline Hipotiroidismo & $3,0 \%$ & $2,3 \%$ & $10,2 \%$ & $7,7 \%$ \\
\hline Lumbago & $6,8 \%$ & $4,1 \%$ & $10,0 \%$ & $6,2 \%$ \\
\hline Demencia & $10,3 \%$ & $7,9 \%$ & $8,5 \%$ & $11,2 \%$ \\
\hline Insuficiencia renal crónica & $11,3 \%$ & $8,8 \%$ & $8,5 \%$ & $6,3 \%$ \\
\hline Insuficiencia cardíaca & $13,0 \%$ & $7,8 \%$ & $9,3 \%$ & $6,9 \%$ \\
\hline Sordera & $5,4 \%$ & $4,0 \%$ & $6,1 \%$ & $4,1 \%$ \\
\hline Asma(tratada actualmente) & $2,9 \%$ & $2,1 \%$ & $5,8 \%$ & $3,7 \%$ \\
\hline $\begin{array}{l}\text { Ceguera y baja visión (excluye trastornos de } \\
\text { refracción) }\end{array}$ & $5,9 \%$ & $4,0 \%$ & $6,6 \%$ & $4,2 \%$ \\
\hline Neuropatía periférica & $4,2 \%$ & $2,7 \%$ & $4,9 \%$ & $3,4 \%$ \\
\hline Divertículos & $4,8 \%$ & $3,3 \%$ & $5,4 \%$ & $3,9 \%$ \\
\hline Gota & $6,7 \%$ & $5,4 \%$ & $1,8 \%$ & $1,2 \%$ \\
\hline Artritis reumatoide y otras & $2,8 \%$ & $2,4 \%$ & $4,5 \%$ & $3,1 \%$ \\
\hline Parkinson & $6,1 \%$ & $3,0 \%$ & $3,7 \%$ & $2,6 \%$ \\
\hline Errores del Metabolismo y Cromosomopatías & $2,3 \%$ & $1,7 \%$ & $1,6 \%$ & $1,2 \%$ \\
\hline Enfermedades crónicas hepatopancreáticas & $2,4 \%$ & $1,6 \%$ & $1,5 \%$ & $1,1 \%$ \\
\hline Enfermedad vascular periférica & $4,8 \%$ & $2,8 \%$ & $1,0 \%$ & $0,7 \%$ \\
\hline Estreñimiento crónico & $3,8 \%$ & $2,1 \%$ & $2,8 \%$ & $2,1 \%$ \\
\hline Distrofia muscular o parálisis & $4,8 \%$ & $1,6 \%$ & $1,9 \%$ & $1,1 \%$ \\
\hline Esquizofrenia & $1,4 \%$ & $0,8 \%$ & $1,2 \%$ & $1,1 \%$ \\
\hline Bronquiectasias & $1,9 \%$ & $1,2 \%$ & $1,1 \%$ & $0,8 \%$ \\
\hline Enfermedades inmunológicas & $1,0 \%$ & $0,8 \%$ & $1,0 \%$ & $0,7 \%$ \\
\hline Síndrome del intestino irritable & $0,7 \%$ & $0,4 \%$ & $1,2 \%$ & $0,8 \%$ \\
\hline
\end{tabular}




\begin{tabular}{lllll}
\hline Epilepsia (tratada actualmente) & $1,4 \%$ & $0,7 \%$ & $0,9 \%$ & $0,6 \%$ \\
\hline Psoriasis o eczema & $1,1 \%$ & $0,8 \%$ & $0,8 \%$ & $0,5 \%$ \\
\hline Hepatitis vírica & $0,7 \%$ & $0,5 \%$ & $0,7 \%$ & $0,5 \%$ \\
\hline Enfermedades Hematológicas crónicas & $1,1 \%$ & $0,7 \%$ & $0,8 \%$ & $0,5 \%$ \\
\hline Abuso de alcohol & $1,1 \%$ & $0,6 \%$ & $0,2 \%$ & $0,1 \%$ \\
\hline Enfermedad inflamatoria intestinal & $0,6 \%$ & $0,5 \%$ & $0,6 \%$ & $0,4 \%$ \\
\hline Sinusitis crónica & $0,4 \%$ & $0,2 \%$ & $0,3 \%$ & $0,2 \%$ \\
\hline Trastornos de Aprendizaje & $0,1 \%$ & $0,1 \%$ & $0,1 \%$ & $0,1 \%$ \\
\hline Migraña & $0,0 \%$ & $0,0 \%$ & $0,2 \%$ & $0,1 \%$ \\
\hline Estado postrasplante & $0,2 \%$ & $0,1 \%$ & $0,1 \%$ & $0,0 \%$ \\
\hline
\end{tabular}

Tabla 3. Porcentaje de la población total del País Vasco (mayor de 65 y de 80 años de edad) incluido en del Servicio Público de Teleasistencia de Euskadi (betiON), en función de su distribución en quintiles de Índice de Privación

\begin{tabular}{lccccc}
\hline & \multicolumn{5}{c}{ Índice de Privación (en quintiles) } \\
\cline { 2 - 6 } & $\mathbf{1}$ & $\mathbf{2}$ & $\mathbf{3}$ & $\mathbf{4}$ & $\mathbf{5}$ \\
\hline Edad>=65 años & & & & & \\
\hline Ambos sexos & $4,7 \%$ & $4,8 \%$ & $5,1 \%$ & $5,3 \%$ & $6,4 \%$ \\
\hline Varones & $2,1 \%$ & $2,4 \%$ & $2,3 \%$ & $2,5 \%$ & $2,9 \%$ \\
\hline Mujeres & $6,5 \%$ & $6,7 \%$ & $7,2 \%$ & $7,5 \%$ & $9,0 \%$ \\
\hline Edad>=80 años & & & & & \\
\hline Ambos sexos & $10,8 \%$ & $11,0 \%$ & $12,0 \%$ & $12,6 \%$ & $14,2 \%$ \\
\hline Varones & $5,9 \%$ & $6,3 \%$ & $6,3 \%$ & $7,0 \%$ & $7,4 \%$ \\
\hline Mujeres & $13,2 \%$ & $13,6 \%$ & $15,2 \%$ & $15,9 \%$ & $18,0 \%$ \\
\hline Nates
\end{tabular}

Nota: Estos grupos no son excluyentes; una persona de más de 80 años de edad está incluida en ambos grupos.

Tabla 4. Porcentajes de personas mayores de 65 años de edad ingresadas una o más veces durante el año 2011 por hospitalizaciones evitables, en función de su sexo y su inclusión o no en el Servicio Público de Teleasistencia de Euskadi (betiON). Odds Ratio ajustadas por edad, índice de privación y comorbilidad (Aggregated Diagnosis Groups)

\begin{tabular}{|c|c|c|c|c|c|}
\hline & \multirow{2}{*}{$\begin{array}{c}\text { Porcentaje de } \\
\text { personas con una o } \\
\text { más hospitalizaciones } \\
\text { evitables }\end{array}$} & \multicolumn{4}{|c|}{ Odds Ratio ajustadas (IC 95\%) } \\
\hline & & \multicolumn{2}{|c|}{$\begin{array}{l}\text { Diferencias } \\
\text { entre sexos }\end{array}$} & \multicolumn{2}{|c|}{$\begin{array}{l}\text { Diferencias entre estar o incluido } \\
\text { o no en teleasistencia }\end{array}$} \\
\hline $\begin{array}{l}\text { Todos los } \\
\text { pacientes }\end{array}$ & $3,14 \%$ & $\begin{array}{l}\text { Varones vs } \\
\text { Mujeres }\end{array}$ & $\begin{array}{c}1,268 \\
(1,215-1,323)\end{array}$ & $\begin{array}{c}\text { Población betiON } \\
\text { vs población Osakidetza }\end{array}$ & $\begin{array}{c}0,975 \\
(0,912-1,043)\end{array}$ \\
\hline Varones & $3,92 \%$ & & & & \\
\hline Mujeres & $2,57 \%$ & & & & \\
\hline \multicolumn{6}{|l|}{ betiON } \\
\hline $\begin{array}{l}\text { Todos los } \\
\text { pacientes }\end{array}$ & $6,94 \%$ & & & & \\
\hline Varones & $11 \%$ & $\begin{array}{l}\text { Varones vs } \\
\text { Mujeres }\end{array}$ & $\begin{array}{c}1,364 \\
(1,185-1,569)\end{array}$ & & \\
\hline Mujeres & $5,96 \%$ & & & & \\
\hline
\end{tabular}


Tabla 5. Promedios de coste en euros de la atención sanitaria proporcionada en un año a las personas incluidas en el Servicio Público de Teleasistencia de Euskadi (betiON). Comparación entre los valores observados y los esperados en función de diferentes ajustes

\begin{tabular}{|c|c|c|c|c|}
\hline & $\begin{array}{c}\text { Ambos } \\
\text { sexos }\end{array}$ & Varones & Mujeres & $\begin{array}{l}\text { Diferencias entre hombres } \\
\text { y mujeres en coste } \\
\text { (ajuste-2) (p_value) }\end{array}$ \\
\hline \multicolumn{5}{|l|}{ Coste total } \\
\hline Observado & 4120,75 & 5211,75 & 3856,91 & \multirow{4}{*}{$<0,0001$} \\
\hline Esperado (ajuste-1) & 2645,55 & 3050,14 & 2547,71 & \\
\hline Esperado (ajuste-2) & 3824,95 & 4671,52 & 3620,23 & \\
\hline Diferencias entre observado y ajuste-2 (p_value) & $<0,0001$ & $<0,0001$ & $<0,0001$ & \\
\hline \multicolumn{5}{|l|}{ Atención Primaria } \\
\hline Observado & 878,28 & 931,82 & 865,33 & \multirow{4}{*}{$<0,0001$} \\
\hline Esperado (ajuste-1) & 600,89 & 593,74 & 602,62 & \\
\hline Esperado (ajuste-2) & 748,34 & 773,86 & 742,17 & \\
\hline Diferencias entre observado y ajuste-2 (p_value) & $<0,0001$ & $<0,0001$ & $<0,0001$ & \\
\hline \multicolumn{5}{|l|}{ Prescripciones } \\
\hline Observado & 747,03 & 836,64 & 725,36 & \multirow{4}{*}{$<0,0001$} \\
\hline Esperado (ajuste-1) & 478,87 & 497,47 & 474,37 & \\
\hline Esperado (ajuste-2) & 591,28 & 640,34 & 579,41 & \\
\hline Diferencias entre observado y ajuste-2 (p_value) & $<0,0001$ & $<0,0001$ & $<0,0001$ & \\
\hline \multicolumn{5}{|l|}{ Especializada sin ingreso } \\
\hline Observado & 585,01 & 654,61 & 568,17 & \multirow{4}{*}{$<0,0001$} \\
\hline Esperado (ajuste-1) & 388,48 & 435,42 & 377,13 & \\
\hline Esperado (ajuste-2) & 547,82 & 651,68 & 522,71 & \\
\hline Diferencias entre observado y ajuste-2 (p_value) & $<0,0001$ & $<0,0001$ & $<0,0001$ & \\
\hline \multicolumn{5}{|l|}{ Urgencias hospital } \\
\hline Observado & 113,81 & 143,4 & 106,65 & \multirow{4}{*}{$<0,0001$} \\
\hline Esperado (ajuste-1) & 72,47 & 78,15 & 71,1 & \\
\hline Esperado (ajuste-2) & 107,37 & 123,34 & 103,51 & \\
\hline Diferencias entre observado y ajuste-2 (p_value) & $<0,0001$ & $<0,0001$ & $<0,0001$ & \\
\hline \multicolumn{5}{|l|}{ Ingresos hospital } \\
\hline Observado & 1796,63 & 2645,27 & 1591,4 & \multirow{4}{*}{$<0,0001$} \\
\hline Esperado (ajuste-1) & 1104,85 & 1445,37 & 1022,5 & \\
\hline Esperado (ajuste-2) & 1830,14 & 2482,3 & 1672,43 & \\
\hline Diferencias entre observado y ajuste-2 (p_value) & $<0,0001$ & $<0,0001$ & $<0,0001$ & \\
\hline
\end{tabular}

Ajuste 1: edad y sexo

Ajuste 2: edad, sexo, índice de privación y Aggregated Diagnosis Groups (ADGs) 


\section{DISCUSIÓN}

Los usuarios del servicio público de teleasistencia del País Vasco presentan una prevalencia más alta de enfermedades crónicas, mayor grado de multimorbilidad y requieren una utilización más elevada de recursos sanitarios que otros ciudadanos del País Vasco de su mismo sexo y edad.

Debido al método de captación, en los pacientes betiON, no se observa un aumento de la multimorbilidad en relación con el envejecimiento. A pesar de sus mayores necesidades de cuidados de salud, una proporción muy importante llevan una vida independiente, casi el $60 \%$ de las mujeres y el $40 \%$ de los varones viven solos. Nuestros análisis no permiten concluir que la inclusión en el programa suponga una disminución de ingresos por hospitalizaciones evitables, pero sí que la calidad de la atención es asimilable a la de la población general. Lo cual es remarcable, ya que La teleasistencia se ha extendido en el País Vasco especialmente entre las poblaciones socialmente más desfavorecidas y en el sexo femenino.

La disponibilidad de la fuente de información creada a partir del proyecto de estratificación poblacional en Euskadi, que conecta diversas bases de datos por medio de un identificador único, permite profundizar en el estudio con una abundancia y calidad de información muy alto.

Un problema que se presenta al obtener prevalencias de enfermedades y multimorbilidad a partir de bases de datos administrativas es el efecto del periodo de seguimiento sobre dichas estimaciones. Por una parte se conoce que la observación de sólo 12 meses puede ser insuficiente, pues incluso en un número significativo de pacientes a los que se ha diagnosticado un problema de salud importante, dicho diagnóstico no se repite en el año siguiente. Por otra parte, un periodo de seguimiento excesivamente largo puede producir una sobreestimación al detectarse enfermedades de curso prolongado, pero que ya no están activas. El uso de prescripciones tampoco está libre de problemas, pues una prescripción aislada no tiene por qué suponer necesaria- mente la presencia de una enfermedad. En nuestro caso, hemos utilizado un método similar al descrito por Barnett y otros ${ }^{14}$, estableciendo criterios específicos para cada una de las 52 enfermedades observadas. Estos criterios varían desde la presencia de un diagnóstico o prescripción aislada, diagnóstico junto con alguna prescripción en el último año, o la presencia repetida en el tiempo de prescripciones o diagnósticos, en función de las características de cada patología.

Nuestro estudio presenta algunas limitaciones. En primer lugar, las bases de datos administrativas sólo pueden contener información sobre morbilidad atendida. Aunque en un sistema de aseguramiento universal como el de Euskadi las barreras a la accesibilidad son menores que en otros lugares, en la medida que determinados subgrupos poblacionales presentan mayores dificultades, sus problemas de salud resultarán peor registrados. Además, en nuestro caso ha resultado factible acceder a variables socioeconómicas registradas correspondientes al área de residencia, pero no a nivel individual. Por otra parte, la estimación de coste de la atención se limita a la utilización de servicios sanitarios y no se incluye la correspondiente a asistencia social. Finalmente, la dimensión calidad de la atención ambulatoria es medida a través de las hospitalizaciones evitables, sin incluir la dimensión de calidad percibida.

Pese a su trascendencia para la investigación médica, la práctica clínica y la organización de la provisión sanitaria y social $^{23,24}$, la multimorbilidad es un fenómeno aún poco estudiado en nuestro medio, tanto en población general como en colectivos específicos. Si bien existen numerosos estudios de prevalencia de multimorbilidad $^{25}$, la ausencia de consenso no sólo sobre cómo definir, sino también sobre las formas de medir estos conceptos, limita la interpretación y la comparabilidad de los resultados ${ }^{26-28}$.

En un momento en que las estrategias de abordaje de la cronicidad se están implementando en el SNS español ${ }^{29}$ resulta necesario ampliar la visión más allá de la atención a las patologías crónicas aisla- 
das $^{30}$, así como generar conocimiento científico relevante en un contexto en el que la investigación médica excluye a los pacientes con multimorbilidad en la mayoría de los ensayos clínicos ${ }^{31}$.

Especialmente en el caso de los ancianos en situación de fragilidad y otras personas con necesidades complejas, el desarrollo de nuevos modelos asistenciales resulta un elemento necesario para conseguir una atención de calidad elevada, universal y sostenible financieramente. En nuestro caso, dado que la población incluida en betiON soporta una mayor carga de cronicidad y multimorbilidad que la población general de sus mismos grupos etarios y, además, requiere una mayor utilización de cuidados sanitarios, se presentan oportunidades de innovación e intervención, como la conexión de bases de datos de carácter sanitario y social; el diseño de programas de gestión de cuidados; y otras intervenciones de coordinación de servicios sanitarios y sociales que reduzcan la utilización global de recursos.

\section{BIBLIOGRAFÍA}

1. Department of Economic and Social Affairs, Population Division, United Nations. World Population Ageing 2009. United Nations.New York; 2009. http://www.un.org/esa/population/ publications/WPA2009/WPA2009_WorkingPaper.pdf. [Fecha de acceso: 2 de mayo de 2013]

2. INE: INEBASE: Padrón municipal de habitantes. Población por edad, sexo y territorio histórico de la CAV. 2010.

3. EUSTAT. Escenarios demográficos 2050. http://www.eustat.es/elementos/ele0001200/ ti_Escenarios_demograficos_2050/ inf0001292_c.pdf. [Fecha de acceso: 2 de mayo de 2013]

4. TinetTi ME, Fried TR, Boyd CM. Designing health care for the most common chronic condition-multimorbidity. JAMA 2012; 307: 2493-2494.

5. Fortin M, Bravo G, Hudon C, Vanasse A, LapoinTE L. Prevalence of multimorbidity among adults seen in family practice. Ann Fam Med 2005; 3: 223-228.

6. Laux G, Kuehlein T, Rosemann T, Szecsenyi J. Coand multimorbidity patterns in primary care based on episodes of care: results from the German CONTENT project. BMC Health Serv Res 2008; 8: 14.
7. Van den AKker M, Buntinx F, Metsemakers JF, Roos S, KNotTnerus JA. Multimorbidity in general practice: prevalence, incidence, and determinants of co-occurring chronic and recurrent diseases. JCE 1998; 51: 367-437.

8. Britt HC, Harrison CM, Miller GC, Knox SA. Prevalence and patterns of multimorbidity in Australia.Med J Aust 2008; 189: 72-77.

9. UiJen AA, Van denLisdonk EH. Multimorbidity in primary care: prevalence and trend over the last 20 years. Eur J Gen Practice 2008; 14: 28-32.

10. Schneider KM, O’Donnell BE, Dean D. Prevalence of multiple chronic conditions in the United States' Medicare population. Health and Qualityof Life Outcomes 2009; 7: 82.

11. Diederichs C, Berger K, Bartels DB. The measurement of multiple chronic diseases-a systematic review on existing multimorbidity indices.J Gerontol A BiolSci Med Sci 2011; 66: 301-311.

12. Lehnert T, Heider D, Leicht H, Heinrich S, Corrieri S, Luppa M, Riedel-Heller S, Konig HH. Review: health care utilization and costs of elderly persons with multiple chronic conditions. Med Care Res Rev 2011; 68: 387-420.

13. Wolff JL, Starfield B, Anderson G. Prevalence, expenditures, and complications of multiple chronic conditions in the elderly.Arch Intern Med 2002; 162: 2269-2276.

14. Barnett K, Mercer SW, Norbury M, Watt G, WyKe S, GuTHRIE B. Epidemiology of multimorbidity and implications for health care, research, and medical education: a crosssectional study. Lancet 2012; 380: 37-43.

15. Glynn LG, Valderas JM, Healy P, Burke E, Newell J, Gillespie P, Murphy AW. The prevalence of multimorbidity in primary care and its effect on health care utilization and cost. FamPract 2011; 28: 516-523.

16. Sancho Castiello M, Del Barrio E, Yanguas LeZAUN J. Estudio sobre la realidad de las personas mayores de 60 años en el País Vasco. Servicio Central de Publicaciones del Gobierno Vasco: Vitoria-Gasteiz; 2011

17. Orueta Jf, Mateos Del Pino M, Barrio Beraza I, Nuño Solinis R, Cuadrado Zubizarreta M, Sola SARABIA C. Estratificación de la población en el País Vasco: resultados en el primer año de implantación; Aten Primaria 2013; 45: 54-60.

18. Caminal J, Mundet X, Ponsà J, Sánchez E, CasanoVA C. Las hospitalizaciones por ambulatorycaresensitiveconditions: selección del listado de códigos de diagnóstico válidos para España. Gac Sanit 2001; 15: 128-141. 
19. Domínguez-Berjón MF, Borrell C, Cano-Serral G, Esnaola S, Nolasco A, Pasarín Mi, Ramis R, SauRina C, Escolar-Pujolar A. Construcción de un índice de privación a partir de datos censales en grandes ciudades españolas: (Proyecto MEDEA). Gac Sanit 2008; 22: 179-187.

20. Registro de Altas de los Hospitales Generales del Sistema Nacional de Salud. CMBD. Norma Estatal. Ministerio de Sanidad, Servicios Sociales e Igualdad. http://www.msc. es/estadEstudios/estadisticas/cmbd.htm. [Fecha de acceso: 2 de mayo 2013]

21. Johns Hopkins University, School of Public Health: The Johns Hopkins University ACG Case-Mix System. http://www.acg.jhsph.org/ index.php?option=com_content\&view=articl e\&id=46\&Itemid=61. [Fecha de acceso: 2 de mayo 2013]

22. Cox DR, Snell EJ. The Analysis of Binary Data, Second Edition, London: Chapman \& Hall. 1989.

23. CAMPBELL-SCHERER D. Multimorbidity: a challenge for evidence-based medicine. Evid Based Med 2010; 15: 165-166.

24. Vogeli C, Shields AE, Lee TA, Gibson TB, Marder WD, WeIss KB et al. Multiple chronic conditions: prevalence, health consequences and implications for quality, care management and costs. J Intern Med 22 (Suppl 3): 391-395.
25. Fortin M, Stewart M, Poitras ME, Almirall J, MadDocks H. A systematic review of prevalence studies on multimorbidity: toward a more uniform methodology. Ann Fam Med 2012; 10: 142-151.

26. Valderas J, Starfield B, Sibbald B, Salisbury C, RoLAND M. Defining comorbidity: Implications for understanding health and health services. Ann Fam Med 2009; 7: 357-363.

27. Starfield B, Kinder K. Multimorbidity and its measurement. Health Policy 2011; 103: 3-8.

28. Huntley AL, Johnson R, Purdy S, Valderas JM, SAlisBury C. Measures of multimorbidity and morbidity burden for use in primary care and community settings: a systematic review and guide. Ann Fam Med 2012; 10: 134-141.

29. García-Goñı M, Hernández-Quevedo C, Nuño-SoLINís R, PAOLuCCI F. Pathways towards chronic care-focused healthcare systems: Evidence from Spain. Health Policy 2012; 108: 236-245.

30. Hughes LD, McMurdo ME, Guthrie B. Guidelines for people not for diseases: the challenges of applying UK clinical guidelines to people with multimorbidity. Age Ageing 2013; 42: 62-69.

31. JADAD AR, To MJ, Emara M, Jones J. Consideration of multiple chronic diseases in randomized controlled trials. JAMA 2011; 306: 26702672 . 